\title{
EL TIPO URBANO Y LAS IDENTIDADES DE LAS CIUDADES \\ LATINOAMERICANAS \\ Perspectivas para su visibilización desde un análisis regional
}

\section{THE URBAN TYPE AND THE IDENTITIES OF LATIN AMERICAN CITIES}

\author{
Perspectives for its visibility from a regional analysis
}

\author{
A. María Rebeca Medina \& B. Silvia Contanzo \& C. María \\ Verónica Cuadrado \& D. Mara G. Carmi \\ Facultad de Arquitectura, Urbanismo y Diseño, Universidad Nacional de Córdoba \\ mrebecamedina@gmail.com \\ arq.silviacostanzo@gmail.com \\ mveronicacuadrado@gmail.com \\ maritacarmignani@hotmail.com
}

\begin{abstract}
RESUMEN
El concepto de "tipo" es frecuentemente utilizado en el estudio de urbano y arquitectónico. En el marco de la posmodernidad nos preguntamos con qué enfoque abordar su estudio para aportar a una planificación urbana sostenible. Esta presentación expone avances de la investigación "Tipo Urbano en América Latina. Una mirada crítica para el diagnóstico urbano sustentable de la región", cuyo tema-problema es la pertinencia de la transferencia de las metodologías extra-regionales en el análisis de tipos urbanos paradigmáticos. La metodología propone elaborar esquemas funcionales urbanos a partir de una lectura gráfica y conceptual, sistematizada y elaborada como continuación de enfoques teóricos previos, por ejemplo M. Santos. Como resultado inicial -ejemplificado en la ciudad de Córdoba-, se detecta a la traza fundacional como la rugosidad que organiza fijos y flujos, los consolida y expande, mientras los actores se movilizan, desplazan, inmigran, migran y emigran, siguiendo la suerte de las inercias del territorio, evidenciando las variantes del tipo urbano latinoamericano.
\end{abstract}

Palabras clave: tipo urbano, esquema urbano, historia urbana, Latinoamérica

Línea de investigación: Ciudad y Proyecto.

Tema: Historia urbana e historia del urbanismo.

\section{ABSTRACT}

In urbanism and architecture studies the notion of 'type' is frequently applied. Within the framework of postmodernity, the research question arises: Which should be the approach to study the 'type' urban to facilitate its transfer to sustainable urban planning? This presentation shows the developments of the research called "Urban Type in Latin America. A critical approach to urban sustainable diagnosis of the countries of the region", whose theme/issue is the relevance of the transfer of extra-regional methodologies in the analysis of 


\section{SÃOPAULO15 $\sim 17 \cdot$ LISBOA $25 \sim 26$ JUN 2020

paradigmatic urban types. The methodology proposes to elaborate urban functional schemes from a graphic and conceptual reading, systematized and elaborated as a continuation of previous theoretical approaches, for example M. Santos. As an initial result of the research -exemplified in the city of Córdoba-, the foundational street grid is detected as the roughness that organizes fixes and flows objects, consolidates and expands them, while the actors mobilize, move, immigrate, migrate and emigrate, following the fate of the inertias of the territory, showing the variants of the Latin American urban type.

\section{Keywords: 'type' urban, urban scheme, urban history, Latin America}

Research line: City and Project.

Topic: Urban history and history of urbanism.

\section{Introducción a la noción de tipo en el estudio urbano}

En el proyecto de investigación sobre el "tipo urbano" en América Latina ${ }^{1}$-del cual se desprende esta presentación-, distintos autores aportaron a la construcción del marco teórico. Por ejemplo, Hardoy (1999) se preguntaba sobre qué es una ciudad en América, y si dicho análisis se podía hacer con iguales metodologías en ciudades europeas como americanas. Si la ciudad posmoderna es desintegrada, fragmentaria, caótica, dispersa, ¿es igual la desintegración en ambos continentes?

Asimismo, aunque en el estudio del urbanismo y de la arquitectura es frecuente utilizar el concepto de "tipo": ¿con qué enfoque se debería abordar su estudio para facilitar su transferencia a la planificación urbana sostenible en la posmodernidad? Construir "tipos urbanos" de referencia para estudiar la ciudad latinoamericana puede permitir generar herramientas de análisis y diseño para aplicar en procesos de planificación sustentables, contemporáneos y futuros.

Tomando el espacio geográfico y cultural latinoamericano, el tema-problema es la verificación de la pertinencia de la transferencia de las metodologías extra-regionales en el análisis de tipos urbanos paradigmáticos en Latinoamérica, para la adaptación y/o propuesta de métodos propios que permitan construir modelos urbanos locales sustentables.

El presente trabajo es un avance de los resultados alcanzados a partir de la construcción de una metodología que se inicia con un relevamiento cartográfico y bibliográfico, para identificar y sistematizar primero casos testigos, y crear un banco de datos cartográficos que contribuya al estudio historiográfico de la forma urbana en Latinoamérica y a la planificación urbana municipal. En segunda instancia, se procede al análisis de la cada ejemplo -en este caso Córdoba-, desde una lectura gráfica y conceptual, elaborada como una instancia

\footnotetext{
${ }^{1}$ La presente ponencia es parte del Proyecto de Investigación "Tipo Urbano en América Latina. Una mirada crítica para el diagnóstico urbano sustentable de la región". Directora: Mgtr. Arq. R. MEDINA, Codirectora: Spec. Arq. S. COSTANZO | Equipo de Trabajo: Integrantes Responsables: Mgtr. Arq. V. CUADRADO, Mgtr. Arq. M. CARMIGNANI | Integrantes colaboradores: Arqs. M. RAME, F. RODRíGUEZ, G. ALCARAZ, S. MANSILLA, C. LAMELAS, P. MOLERO, C. RASINO, L. ESQUIBEL, L. AIMARETTI, J. TOSCANO; Adscriptos Alumnos: S. MUTIGLIENGO, V. KARL | Integrantes Colaboradores externos: Mgtr. Arq. C. TORTONE (UM), Esp. Arq. M. GRANIZO, Arqs. E. ACUÑA, M. BARRIONUEVO, E. AREVALO. Proyecto Consolidar SECYT, UNC. 2018-2021. En el Primer Informe de avance se trabajaron ciudades prehispánicas (Caral, Tikal, Machu Pichu, Teotihuacán) y coloniales (Tilcara, El Shincal de Quimivil, La Antigua, Cartagena de Indias, Trujillo, Potosí, Quito, Córdoba, Misión de San Ignacio, Santa Fe La Vieja, Puebla), graficando los patrones propuestos por K. Lynch, P. Panerai, R. Krier, Ch. Alexander, M. Santos.

El enfoque propuesto por M. Santos específicamente fue aplicado en la Primera Fase del Plan Maestro de La Antigua Guatemala (UNESCO-MCD, 2015), Villa del Tránsito-Villa Cura Brochero (ICOMOS Argentina, 2018), entre otras, y al desarrollo de los contenidos del Módulo: Historia del Paisaje y la Cultura, Especialidad en Planificación y Diseño del Paisaje, FAUD-UNC, desde 2010.
} 


\section{XII $\square$ SÃOPAULO15 17 LISBOA $25 \sim 26$ JUN 2020}

de continuidad de enfoques teóricos previos -como los de Hardoy, Panerai, Krier, Cerasi, Linch, Alexander, Santos-, pero desde una "aproximación" latinoamericana, hasta llegar a la elaboración gráfica del "tipo urbano".

Retomando el concepto de "tipo", J. Ferrater Mora (1951) (1951:793), proponía que "el tipo puede referirse tanto a uno de los ejemplares concretos que manifiesta o representa con claridad eminente su clase (modelo, arquetipo), como al conjunto de determinaciones cuyo carácter está impreso en todos los ejemplares correspondientes (prototipo, tipo ideal). El "tipo" es un esquema representativo, la forma que proporciona una imagen de acuerdo con la cual los demás ejemplares están hechos". No se trata de generar modelos, sino un esquema que sirva de regla al modelo, que permita análisis, comparaciones, aplicaciones y transferencias.

Si la forma urbana es la conformación exterior y global de la ciudad, resultado de la disposición de sus elementos constitutivos, de las interacciones establecidas entre ellos, el soporte físico-natural y la cosmovisión construida socialmente, ¿cómo es la forma urbana de la ciudad latinoamericana? ¿resultado de qué? ¿Puede el estudio del "tipo urbano" aportar en un proceso de planificación sustentable contemporáneo?

A. C. Quatrémère de Quincy (2007: 240) (1792-1825), plantea el "tipo" como la idea de un elemento que debe servir de regla y no la imagen a imitar: "la palabra tipo no representa tanto la imagen de una cosa que copiar o que imitar perfectamente, cuanto la idea de un elemento que debe servir de regla al modelo (...), el tipo es un objeto según el cual nadie puede concebir obras que no se asemejen en absoluto entre ellas. Todo es preciso y dado en el modelo; todo es más o menos vago en el tipo".

Desde este enfoque, construir "tipos urbanos" de referencia para el estudio de la ciudad latinoamericana, incluyendo todas sus etapas históricas, que consideren la relación región-cultura, que interpreten la cartografía histórica y la gráfica digital, puede generar herramientas de análisis y diseño aplicables en los procesos de planificación sustentables contemporáneos y futuros.

En relación con la construcción del enfoque teórico, hablar de gestión y desarrollo sostenible desde la historia es una necesidad ante el complejo y diverso escenario actual. Miradas que deben ser transversales, como la del geógrafo y abogado Milton Santos, (1926-2001) (1996). Su enfoque teórico desde la Geografía Humana (2000), plantea otra mirada sobre los patrones históricos de uso del suelo, que permiten orientar y fundamentar una planificación urbana y territorial sustentable. Para Santos, el análisis territorial de un sitio cultural debe centrarse en la identificación de los elementos que manifiestan la particularidad del territorio, y constituyen su soporte fundamental al definir identidades y memorias colectivas.

De acuerdo a Santos (2000) la noción de espacio se entiende como aquel conjunto indisoluble, solidario y también contradictorio de sistemas de objetos y sistemas de acciones, que deben ser considerados en el contexto único histórico. Para analizarlo, propone los conceptos de:

- Fijos: elementos fijados en cada lugar, que permiten sobre ellos acciones que lo modifican.

- Rugosidades: es lo que permanece del pasado como forma, como espacio construido, como paisaje, lo que resta de un proceso de acumulación, supresión, superposición a través del cual las cosas se sustituyen y acumulan en cada lugar. Las rugosidades suman los testimonios de diferentes momentos históricos, que permanecen o mutan -parcial o totalmente-, de acuerdo a las nuevas funciones, separando forma y contenido, con nuevos significados y valores. Este cierto carácter estable se relaciona con los fijos. 


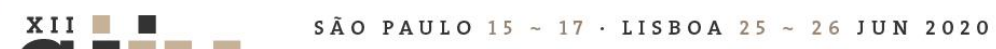

- Flujos: son el resultado directo de las acciones y atraviesan o se instalan en los fijos, modificando su significación y su valor, al mismo tiempo que ellos también se modifican.

- Inercias Dinámicas: el espacio organizado o las formas espaciales, si bien son rugosidades son también inercia dinámica, es decir, el resultado de la interacción de múltiples variables presentes que ejercen condicionamientos o determinaciones sobre los procesos futuros.

Comparado con otros autores trabajados, es Santos quien, a nuestro criterio, más aporta en la evolución y construcción de metodologías de análisis urbano más críticos, que superen la descripción en búsqueda de la interpretación, como continuidad de lo previamente indagado, y no como moda efímera sin contenidos.

A. continuación, se desarrolla un caso de estudio -Córdoba-, bajo los componentes de análisis propuestos por M. Santos ${ }^{2}$.

\section{Caso de Estudio: Córdoba. Enfoque teórico para elaborar un modelo}

Relacionando características similares propuestas por Santos (2000), se agruparon conceptos por sus referencias a los objetos y sus relaciones en el espacio: rugosidades e inercias contribuyen a definir la profundidad histórica del territorio y su identidad, que se expresa en el patrimonio territorial (ambiental, construido, el socioeconómico, cultural y social).

En referencia al sitio, Córdoba ha sido analizada desde múltiples variables y enfoques. En este caso, se parte de registros gráficos para definir siete etapas de desarrollo sobre el territorio, para identificar fijos y flujos, rugosidades e inercias dinámicas, y construir otra mirada sobre su patrimonio tangible e intangible.

\footnotetext{
2 En el desarrollo de la investigación se elaboraron análisis gráficos de las etapas históricas de la ciudad según: Kevin Lynch [hitos, zonas/barrios, bordes, nodos, sendas], Philippe R. Panerai [la parcela, la manzana, la trama], Rob Krier [llenos /vacíos: calle/plaza/manzana], Christopher Alexander [niveles de escala, repetición, simetrías locales, ecos, no separación], Milton Santos [fijos rugosidades, flujos, inercias dinámicas]. La complejidad de comparar la producción gráfica condujo a la definición de selección de etapas clave para enfocar el análisis, y a ejemplificar con una ciudad en esta presentación.
} 


\section{SÃO PAULO15 17 LISBOA $25 \sim 26$ JUN 2020}

\section{El territorio de la ciudad de Córdoba desde el enfoque de Milton Santos}

\subsection{Asentamientos prehispánicos}

La Tabla 01 sistematiza los datos del territorio en esta etapa histórica según los componentes de análisis indicados.

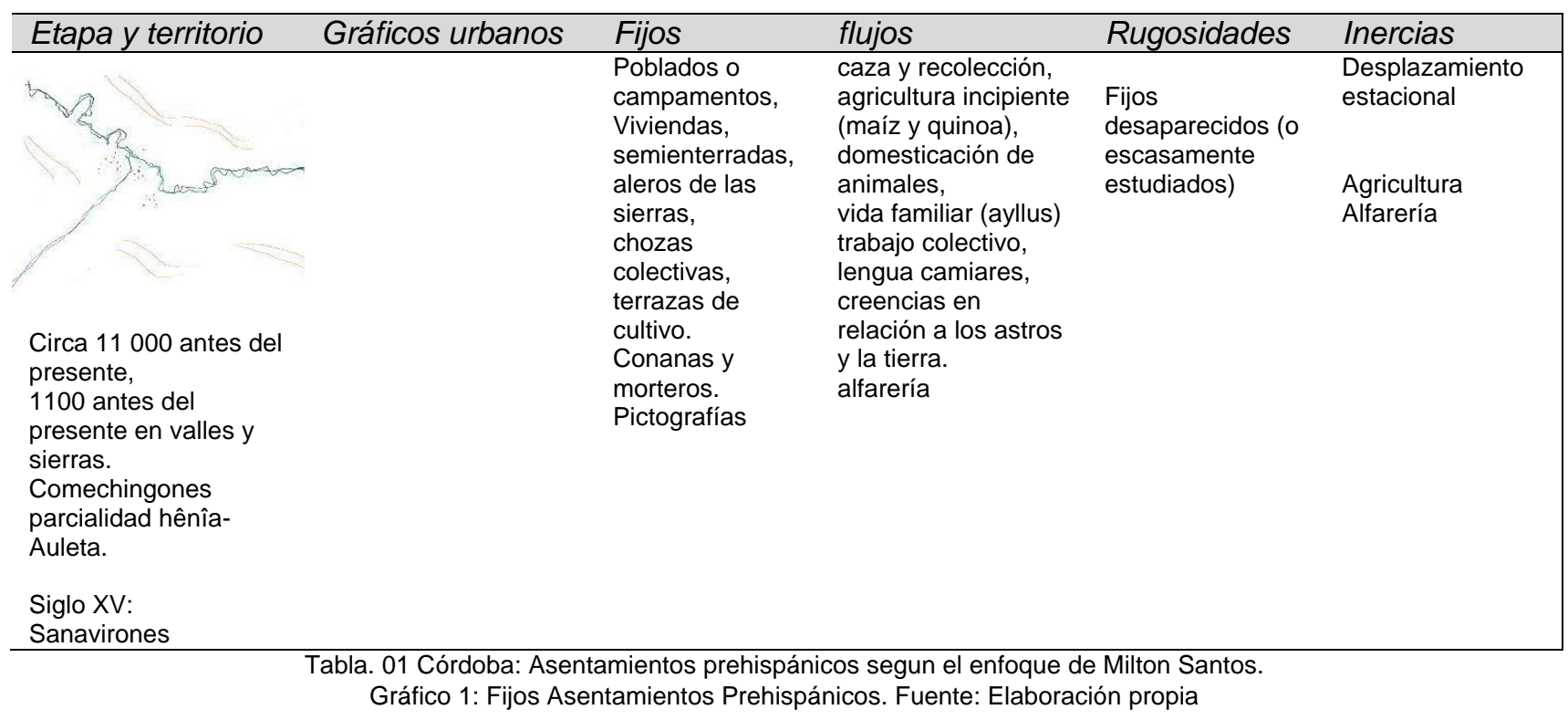

Los asentamientos de los comechingones y luego sanavirones significaron fijos no permanentes, que dieron marco al nomadismo de grupos entre el valle y la sierra. Sus artefactos dejaron testimonio de sus flujos, mientras sus fijos desaparecieron con el tiempo. Las rugosidades y las inercias que dejaron son, en su mayoría, de carácter inmaterial.

Esta fragilidad de fijos y rugosidades arquitectónicas y urbanas generó su marginación al momento de reflexionar sobre su participación en el espesor del territorio, no ocupando un lugar destacado como antecedente.

Se destacada en cambio la relación entre españoles que llegaron al Quisquisacate y sus pobladores sanavirones, originando la mixogenización inicial de la población, una inercia dinámica que luego se afianzaría en el territorio. 


\section{SÃO PAULO15 17 LISBOA $25 \sim 26$ JUN 2020}

Seminário Internacional de Investigação em Urbanismo

Seminario Internacional de Investigación en Urbanismo

\subsection{Primera fundación de la ciudad, 1573}

La Tabla 02 sistematiza los datos del territorio en esta etapa histórica según los componentes de análisis indicados.

\begin{tabular}{|c|c|c|c|c|c|}
\hline Etapa y territorio & Gráficos urbanos & Fijos & flujos & Rugosidades & Inercias \\
\hline $\begin{array}{l}\text { Corriente colonizadora } \\
\text { del Norte. } \\
\text { 1528. Francisco César } \\
\text { 1545. Francisco de } \\
\text { Mendoza } \\
\text { 1549-1782. } \\
\text { Gobernación del } \\
\text { Tucumán/Provincia } \\
\text { del Tucumán, Juríes y } \\
\text { Diaguitas } \\
\text { 1556. Francisco de } \\
\text { Aguirre } \\
1573 \text {. Jerónimo Luis } \\
\text { de Cabrera } \\
\text { Primeros vecinos } \\
\text { (comitiva del fundador, } \\
80 \text { personas) }\end{array}$ & & $\begin{array}{l}\text { Poblado } \\
\text { sanavirones } \\
\text { (Quisquisacate) } \\
\text { Fundación de } \\
\text { Córdoba de la } \\
\text { Nueva } \\
\text { Andalucía. } \\
\text { Fuerte, } \\
\text { Viviendas. } \\
\text { Río, arroyo y } \\
\text { barrancas }\end{array}$ & $\begin{array}{l}\text { Exploración del } \\
\text { territorio (salida al } \\
\text { mar). } \\
\text { Conquista y } \\
\text { apropiación. } \\
\text { Evangelización: } \\
\text { religión católica } \\
\text { Idioma castellano. }\end{array}$ & $\begin{array}{l}\text { Desaparecidas. } \\
\text { Fijos } \\
\text { abandonados, } \\
\text { condicionados } \\
\text { por la topografía }\end{array}$ & $\begin{array}{l}\text { Movilidad. } \\
\text { Control del } \\
\text { territorio } \\
\text { Religión católica }\end{array}$ \\
\hline
\end{tabular}

Tabla. 02 Córdoba: Primera Fundación según Milton Santos. Gráfico 2: Fijos Primera Fundación. Fuente: Elaboración Propia

El avance de la conquista y ocupación del territorio supuso uno de los principales flujos, marcado por el interés en rugosidades míticas o prácticas, como la salida al mar.

Los conquistadores se movilizaron desde el Norte, buscando sitios de asentamiento para fundar nuevas ciudades. Encontraron un curso de agua (Río Suquía) y barrancas: estos fijos determinaron una topografía favorable para establecerse. Esta localización duró poco tiempo y los nuevos fijos (fuerte y otros), no dejaron rastros.

Aunque los fijos suelen ser efímeros en esta época, comienzan a destacarse fijos de naturaleza geográfica el río, el arroyo y las barracas-, que condicionaron la construcción de una nueva rugosidad. El flujo más importante, además del político y administrativo y el idioma que imponen los conquistadores, es la religión católica que inicia su accionar en el territorio. 


\section{SÃO PAULO15 17 LISBOA $25 \sim 26$ JUN 2020}

Seminário Internacional de Investigação em Urbanismo

Seminario Internacional de Investigación en Urbanismo

\subsection{Segunda fundación de la ciudad, $1577-1770$}

La Tabla 03 sistematiza los datos del territorio en esta etapa histórica según los componentes de análisis indicados.

\begin{tabular}{|c|c|c|c|c|c|}
\hline Etapa y territorio & Gráficos urbanos & Fijos & flujos & Rugosidades & Inercias \\
\hline $\begin{array}{l}\text { 1577. traslado de la } \\
\text { fundación por } \\
\text { Lorenzo Suárez de } \\
\text { Figueroa } \\
\text { 1613. Universidad } \\
\text { 1623. Aduana Seca } \\
\text { 1760. primer censo }\end{array}$ & & $\begin{array}{l}\text { Río, arroyo y } \\
\text { barrancas. } \\
\text { Plano fundacional: } \\
\text { Traza. 7x10 } \\
\text { manzanas } \\
\text { Plaza mayor, } \\
\text { Sedes } \\
\text { administración: } \\
\text { Cabildo, } \\
\text { Aduana. } \\
\text { Sedes religiosas: } \\
\text { Catedral. } \\
\text { Conventos urbanos } \\
\text { (San Francisco, } \\
\text { Jesuitas, Santo } \\
\text { Domingo, La } \\
\text { Merced). } \\
\text { Universidad. } \\
\text { Viviendas (solares) } \\
\text { y ranchos. }\end{array}$ & $\begin{array}{l}\text { Residencia. } \\
\text { Comercio (Alto } \\
\text { Perú). } \\
\text { Nuevos vecinos } \\
\text { españoles, criollos } \\
\text { Esclavizados. } \\
\text { Enseñanza } \\
\text { universitaria. } \\
\text { Religión católica. } \\
\text { Administración } \\
\text { colonial. }\end{array}$ & $\begin{array}{l}\text { Trazado } \\
\text { fundacional sin } \\
\text { superar barreras } \\
\text { naturales. } \\
\text { Universidad. } \\
\text { Administración. }\end{array}$ & $\begin{array}{l}\text { Religión. } \\
\text { Educación } \\
\text { superior. } \\
\text { Comercio. } \\
\text { Administración } \\
\text { territorial } \\
\text { colonial. }\end{array}$ \\
\hline
\end{tabular}

Los conquistadores desplazaron la fundación urbana hacia el Sur del río, sitio que estaba rodeado por barrancas al Sud y un arroyo al Oeste, buscando mejor localización. Este asentamiento fue el definitivo, y nuevos fijos y flujos se fueron construyendo sobre el plano de fundación. Esto determinó el arraigo de la población que fue interactuando con las rugosidades (Cabildo, Catedral, Universidad, Aduana), y consolidaron la territorialidad en esta etapa.

Reubicados los primero fijos, su relación con el territorio se modifica, y a partir de los flujos -administración, residencia, religión-, lentamente se construyen y consolidan nuevos fijos. Algunos fijos alcanzan jerarquías que identifican a las rugosidades cordobesas: la religión y el accionar de las órdenes católicas, en especial los jesuitas. Se inician, así, las inercias dinámicas históricas: la religión y la educación superior. 


\section{SÃO PAULO15 17 LISBOA $25 \sim 26$ JUN 2020}

Seminário Internacional de Investigação em Urbanismo

Seminario Internacional de Investigación en Urbanismo

\subsection{Consolidación de la traza fundacional, 1770-1870}

La Tabla 04 sistematiza los datos del territorio en esta etapa histórica según los componentes de análisis indicados.

\begin{tabular}{|c|c|c|c|c|c|}
\hline Etapa y territorio & Gráficos urbanos & Fijos & flujos & Rugosidades & Inercias \\
\hline $\begin{array}{l}\text { 1767. Expulsión de los } \\
\text { jesuitas. } \\
\text { 1776. Virreinato del } \\
\text { Rio de la Plata. } \\
\text { 1785. Intendencia de } \\
\text { Córdoba del Tucumán } \\
\text { Rafael de Sobremonte } \\
\text { 1810. Revolución de } \\
\text { Mayo. } \\
\text { 1815. Primer } \\
\text { gobernador. } \\
\text { 1816. } \\
\text { Independencia } \\
\text { Nacional. } \\
\text { 1853. Constitución } \\
\text { Nacional. } \\
\text { 1857. Municipalidad }\end{array}$ & & $\begin{array}{l}\text { Quintas. } \\
\text { Puentes sobre } \\
\text { arroyo La } \\
\text { Cañada. } \\
\text { Densificación } \\
\text { residencias. } \\
\text { Universidad } \\
\text { (franciscanos, } \\
\text { Provincia, } \\
\text { nacionalización). }\end{array}$ & $\begin{array}{l}\text { Reformas } \\
\text { administrativas. } \\
\text { Ordenanzas de } \\
\text { Sobremonte: } \\
\text { urbanas, gremios. } \\
\text { Residencia. } \\
\text { Comercio. } \\
\text { Estudios superiores. } \\
\text { Religión católica. } \\
\text { Contrarrevolución. } \\
\text { Inestabilidad política } \\
\text { (unitarios). }\end{array}$ & $\begin{array}{l}\text { Consolidación y } \\
\text { densificación, } \\
\text { residencias. } \\
\text { Trazado } \\
\text { fundacional } \\
\text { consolidado sin } \\
\text { superar barreras } \\
\text { naturales. }\end{array}$ & $\begin{array}{l}\text { Caudillos } \\
\text { locales. } \\
\text { Centro político y } \\
\text { religioso con } \\
\text { educación } \\
\text { superior. } \\
\text { Oposición al } \\
\text { centralismo } \\
\text { porteño. }\end{array}$ \\
\hline
\end{tabular}

Este período evidencia la aparición de nuevos fijos, que aportan a la consolidación fundacional, surgiendo las quintas hacia el Oeste de la ciudad. Con la nueva estructura administrativa virreinal, sufre modificaciones la relación con el puerto de Buenos Aires: se afianza la ciudad como un centro.

Un flujo eficiente -el jesuita-, es expulsado del sistema, abandonando los fijos, que no recuperan la dinámica original, resintiendo la inercia general territorial. La intención de reestructurar los flujos administrativos genera cierta densificación en los fijos urbanos, pero ésta permanece dentro de los límites de la traza fundacional en todo el periodo, marcando crecimiento hacia el oeste, de uso semi-rural.

Posterior al gobierno de Sobremonte, hay cambios inestables en los flujos políticos que afectan las inercias de educación, religión y política. El flujo de la Universidad se constituye en el elemento más significativo, que influyen en las inercias dinámicas, como un polo político-administrativo que competirá con el poder central de Buenos Aires.

En un marco de inestabilidad política general en el territorio nacional, las rugosidades y las inercias dinámicas -e históricas-, locales, definen y señalan su propia identidad cordobesa y federal. 


\section{SÃOPAULO15 17 LISBOA $25 \sim 26$ JUN 2020}

Seminário Internacional de Investigação em Urbanismo

Seminario Internacional de Investigación en Urbanismo

\subsection{Barrios-pueblos, $1880-1930$}

La Tabla 05 sistematiza los datos del territorio en esta etapa histórica según los componentes de análisis indicados.

\begin{tabular}{|c|c|c|c|c|c|}
\hline Etapa y territorio & Gráficos urbanos & Fijos & flujos & Rugosidades & Inercias \\
\hline $\begin{array}{l}\text { Generación del '80. } \\
1870 \text {. Loteos para los } \\
\text { barrios-pueblo. } \\
\text { 1876. Ley Avellaneda } \\
\text { (inmigración). } \\
\text { 1911. Ley Sáez Peña } \\
\text { Inmigración europea } \\
\text { y migración interna. } \\
\text { 1876. FFCC. }\end{array}$ & 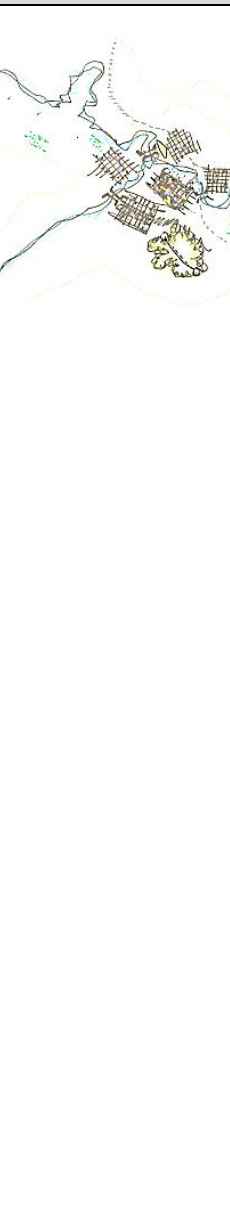 & $\begin{array}{l}\text { Barrios Gral. Paz, } \\
\text { San Vicente, } \\
\text { Pueblo Nuevo, } \\
\text { Nueva Córdoba, } \\
\text { Alberdi, Alta } \\
\text { Córdoba, San } \\
\text { Martin. } \\
\text { Escuelas públicas } \\
\text { y privadas, } \\
\text { Bancos, teatros, } \\
\text { cárcel, FFCC, } \\
\text { administración } \\
\text { (justicia), } \\
\text { cementerio, } \\
\text { mercados, } \\
\text { Hospitales. } \\
\text { Observatorio } \\
\text { Astronómico, } \\
\text { Academia } \\
\text { Nacional de } \\
\text { Ciencias } \\
\text { (historicismo } \\
\text { académico). } \\
\text { Parques y plazas } \\
\text { urbanas, } \\
\text { Residencias. } \\
\text { Modificaciones } \\
\text { urbanas (modelo } \\
\text { Haussmann). } \\
\text { Infraestructura y } \\
\text { equipamiento } \\
\text { urbano } \\
\text { (alumbrado, agua, } \\
\text { pavimento, tranvía) } \\
\text { Fábricas, Molinos. }\end{array}$ & $\begin{array}{l}\text { Inmigración } \\
\text { europea } \\
\text { (construcción mano } \\
\text { de obra). } \\
\text { Comercio: modelo } \\
\text { agroexportador. } \\
\text { Educación pública } \\
\text { y privada. } \\
\text { Reforma educativa. } \\
\text { Administración } \\
\text { pública. } \\
\text { Consolidación, } \\
\text { densificación y } \\
\text { jerarquización de } \\
\text { residencias, } \\
\text { algunas de ellas } \\
\text { estacionales. }\end{array}$ & $\begin{array}{l}\text { Expansión de la } \\
\text { traza } \\
\text { fundacional, } \\
\text { superando } \\
\text { barreras } \\
\text { naturales: } \\
\text { barrios-pueblo. } \\
\text { Sedes } \\
\text { administrativas y } \\
\text { educativas } \\
\text { monumentales. } \\
\text { Trazado FFCC. }\end{array}$ & $\begin{array}{l}\text { Educación } \\
\text { superior, } \\
\text { primaria y } \\
\text { secundaria, } \\
\text { Reforma } \\
\text { educativa. } \\
\text { Consolidación } \\
\text { política a nivel } \\
\text { nacional. }\end{array}$ \\
\hline
\end{tabular}

Tabla. 05 Córdoba: Barrios-Pueblos segun el enfoque de Milton Santos. Gráfico 5: Fijos Barrios-Pueblos.

Fuente: Elaboración propia

La ciudad supera sus fijos naturales, expandiéndose hacia los nuevos fijos que significan los barrios-pueblo, superando los límites naturales y modificando, en parte, las rugosidades (barrancas, río y arroyo). También la traza del ferrocarril se incorpora a la ciudad, y con él la inmigración será el flujo que determina la conformación y la imagen urbana, asociado a la consolidación, densificación y jerarquización de residencias.

Nuevos flujos políticos administrativos atraviesan los fijos existentes, y crean otros tantos más, consolidando las rugosidades heredadas. Los flujos de infraestructura sirven, conectan y vinculan el centro de la ciudad y sus barrios, que diversifican sus flujos tradicionales de residencia y educación. 


\section{SÃOPAULO15 17 LISBOA $25 \sim 26$ JUN 2020 \\ Seminário Internacional de Investigação em Urbanismo \\ Seminario Internacional de Investigación en Urbanismo}

Los fijos aumentan en cantidad y jerarquía, especialmente en educación y ciencia, reafirmando la especialidad de la rugosidad. Otras formas de producción industrial se agregan como flujos e inercias dinámicas, acompañados por la mano de obra inmigrante que ingresa al territorio.

La inercia dinámica propia de la generación del ' 80 y del modelo agroexportador encuentran en Córdoba un territorio que adopta fácilmente nuevos fijos y flujos y las inercias que generan, aportando mayor complejidad a la rugosidad, que consigue mantener su especialidad y consolidar su identidad.

La Reforma Universitaria de 1918 es un flujo local propio de la rugosidad cordobesa, que modifica fijos y flujos propios, pero como inercia dinámica alcanza repercusión a escala latinoamericana.

\subsection{Industrialización y primer ordenamiento urbano, 1930-1980}

La Tabla 06 sistematiza los datos del territorio en esta etapa histórica según los componentes de análisis indicados.

\begin{tabular}{|c|c|c|c|c|}
\hline Etapa y territorio Gráficos urbanos & Fijos & flujos & Rugosidades & Inercias \\
\hline $\begin{array}{l}\text { 1927. Fábrica Militar } \\
\text { de Aviones. } \\
\text { 1936. Gobierno de } \\
\text { Sabattini. } \\
\text { 1946. PJ, } \\
\text { Intervenciones } \\
\text { federales. } \\
\text { 1952.EPEC } \\
\text { 1953. UTN } \\
\text { 1955. IKA } \\
\text { 1956. UCC } \\
\text { 1960. FIAT } \\
\text { 1960. Canal 10 } \\
\text { 1961. Canal 12 } \\
\text { 1969. Cordobazo } \\
\text { 1971. Viborazo, } \\
\text { Gobiernos de facto } \\
\text { 1978. Subsede } \\
\text { Mundial de Fútbol }\end{array}$ & $\begin{array}{l}\text { Escuelas Sabattini, } \\
\text { Colegio Manuel } \\
\text { Belgrano, } \\
\text { (racionalismo y } \\
\text { neocolonial). } \\
\text { Palacio de Justicia. } \\
\text { Fábricas, } \\
\text { Encauzamiento de } \\
\text { La Cañada. } \\
\text { Sociedades } \\
\text { Mutuales y clubes } \\
\text { deportivos. } \\
\text { Hospitales. } \\
\text { Ciudad } \\
\text { Universitaria, UNC. } \\
\text { Palacio } 6 \text { de Julio. } \\
\text { Barrios obreros, } \\
\text { Santa Isabel, } \\
\text { Ferreyra. } \\
\text { Ampliación de } \\
\text { avenidas. } \\
\text { 1970. Primera } \\
\text { peatonalización. } \\
\text { Sustitución de } \\
\text { plazas. } \\
\text { Estadio Chateau } \\
\text { Carreras. }\end{array}$ & $\begin{array}{l}\text { Migración } \\
\text { (alumnos). } \\
\text { Comercio: inicio y } \\
\text { desarrollo de la } \\
\text { industria nacional } \\
\text { (metalmecánica). } \\
\text { Declaratoria MHN, } \\
\text { fijos coloniales. } \\
\text { Planificación } \\
\text { urbana: } \\
\text { 1927. Plan } \\
\text { Carrasco. } \\
\text { 1954. Plan La } \\
\text { Padula. } \\
\text { Educación. } \\
\text { Medicina-sanidad. } \\
\text { Actividad gremial. } \\
\text { Protestas obreras } \\
\text { y estudiantiles. }\end{array}$ & $\begin{array}{l}\text { Nuevas sedes } \\
\text { administrativas y } \\
\text { educativas } \\
\text { monumentales. } \\
\text { Red vial. } \\
\text { Destrucción de } \\
\text { fijos de valores } \\
\text { culturales } \\
\text { modestos. } \\
\text { MHN coloniales. }\end{array}$ & $\begin{array}{l}\text { Industrialización, } \\
\text { Producción } \\
\text { metalmecánica, } \\
\text { Nuevas ofertas } \\
\text { en educación. } \\
\text { Tecnología. } \\
\text { Inicio de la } \\
\text { planificación } \\
\text { urbana. } \\
\text { Renovación } \\
\text { urbana en el } \\
\text { centro histórico. } \\
\text { Polo sanitario } \\
\text { especializado. }\end{array}$ \\
\hline
\end{tabular}

Tabla. 06 Córdoba: Industrialización segun el enfoque de Milton Santos. Gráfico 6: Fijos Industrialización.

Fuente: Elaboración propia

La ciudad se consolida y densifica, incorporando nuevos fijos que le suman un carácter industrial y tecnológico: fábricas de aviones, vehículos y otros, transformarán en zonas industriales a áreas periféricas y vacantes. Ello llevará consigo la radicación de barrios obreros en sus entornos. Destacamos, entre los flujos, las protestas de obreros y estudiantes, que provienen de fijos existentes y nuevos (universidad y fábricas). 


\section{SÃOPAULO15 17 LISBOA 25 26 JUN 2020 \\ Seminário Internacional de Investigação em Urbanismo \\ Seminario Internacional de Investigación en Urbanismo}

El periodo registra el inicio de la planificación de fijos y flujos urbanos, aumentando servicios e infraestructura que modifican y/o sustituyen antiguos fijos. Como resultado, la ciudad pierde fijos de valor cultural modesto, y suma fijos a escala territorial que adquieren valor cultural, definiendo la imagen simbólica de la rugosidad. En contraposición, sus fijos culturales más antiguos son reconocidos por la Nación, sin relación a los flujos que alojaron.

Una modificación fundamental de la rugosidad será la peatonalización de tramos de calles del Centro Histórico, que va a modificar los flujos y posteriormente se constituirá en una rugosidad urbana identitaria.

El proceso de industrialización como inercia dinámica reúne múltiples flujos que atraviesan diversas escalas urbanas y sociales. La industria metalmecánica es el flujo que desarrolla la inercia dinámica del territorio. En correspondencia, el flujo educativo se especializa en tecnología, y adopta nuevos tipos funcionales y lenguajes -a veces monumentales-, para sus fijos.

Nuevamente hay cambios inestables de los flujos políticos, que afectan la inercia de la producción y la educación, organizando, en contrapartida, protestas obreras y estudiantiles, que como flujos modifican fijos locales, pero como inercias alcanzan repercusión nacional.

\subsection{Globalización y segundo ordenamiento urbano, 1980 al presente}

La Tabla 07 sistematiza los datos del territorio en esta etapa histórica según los componentes de análisis indicados.

\begin{tabular}{|c|c|c|c|c|c|}
\hline Etapa y territorio & Gráficos urbanos & Fijos & flujos & Rugosidades & Inercias \\
\hline $\begin{array}{l}\text { 1983. Retorno a la } \\
\text { democracia. } \\
\text { Retroceso industrial. } \\
\text { 1991. Privatización } \\
\text { FFCC. } \\
\text { Nuevo complejo FIAT } \\
\text { 1996. } \\
\text { 2001. Crisis } \\
\text { económica. } \\
\text { 2004. recuperación } \\
\text { económica, } \\
\text { PyMES, } \\
\text { Polo industrial. }\end{array}$ & & $\begin{array}{l}\text { Centros Culturales } \\
\text { (mercados). } \\
\text { Centros de } \\
\text { Participación } \\
\text { Comunal (9). } \\
\text { Peatonalización } \\
\text { área central. } \\
\text { Galerías. } \\
\text { Propiedad } \\
\text { horizontal. } \\
\text { Escuelas } \\
\text { primarias, } \\
\text { Universidades } \\
\text { privadas. } \\
\text { Hospitales. } \\
\text { Nuevas sedes } \\
\text { administrativas. } \\
\text { Barrios privados. } \\
\text { Shoppings. } \\
\text { Nudos urbanos. } \\
\text { Polos industriales. } \\
\text { Terminales de } \\
\text { transporte aéreas } \\
\text { y terrestre. }\end{array}$ & $\begin{array}{l}\text { Descentralización } \\
\text { administrativa. } \\
\text { Migración } \\
\text { (alumnos). } \\
\text { comercio } \\
\text { internacional. } \\
\text { regulación urbana: } \\
\text { ordenanzas } \\
\text { municipales: } \\
\text { 8056,8057, 8060, } \\
\text { 8048, 8133. } \\
\text { Declaratoria } \\
\text { UNESCO } \\
\text { Manzana y } \\
\text { Estancias } \\
\text { Jesuíticas de } \\
\text { Córdoba. }\end{array}$ & $\begin{array}{l}\text { Densificación de } \\
\text { la traza } \\
\text { fundacional. e } \\
\text { inicio de la } \\
\text { sustitución del } \\
\text { tejido urbano de } \\
\text { los barrios- } \\
\text { pueblo. } \\
\text { Reestructura- } \\
\text { ción vial. }\end{array}$ & $\begin{array}{l}\text { Polo sanitario de } \\
\text { alta complejidad. } \\
\text { Turismo ¿cultural? } \\
\text { internacional y } \\
\text { local. } \\
\text { Renovación y } \\
\text { sustitución urbana } \\
\text { en los barrios } \\
\text { tradicionales. } \\
\text { Diversificaciones } \\
\text { en la educación } \\
\text { superior. }\end{array}$ \\
\hline
\end{tabular}




\section{Siliu. \\ SÃO PAULO $15 \sim 17$. LISBOA $25 \sim 26$ JUN 2020 \\ Seminário Internacional de Investigação em Urbanismo \\ Seminario Internacional de Investigación en Urbanismo}

Este período está signado por la economía, conllevando a una serie de modificaciones, ampliaciones, reconversiones -los barrios privados serán la demostración de ello-. También shoppings y grandes centros comerciales que se localizan en barrios-pueblos o de mayor jerarquía.

A partir de esta etapa, se acelera la dinámica de cambio y la renovación por sustitución del tejido urbano: en los barrios-pueblo, por densificación e inserción de edificios en altura; en otros casos, resurgen zonas deprimidas, con nuevos usos.

La administración municipal planifica la rugosidad urbana con nuevos intereses, avanzando en la elaboración de regulaciones diversas, que incluyen fijos culturales, algunos de los cuales serán reconocidos a escala municipal, nacional e internacional.

La fluctuación irregular de los flujos políticos y económicos marca cíclicamente estas décadas. Paralelamente al crecimiento de la traza urbana, los fijos y rugosidades tradicionales son aislados, modificados, resignificados, reciclados o sustituidos. Siguiendo la influencia internacional y globalizada, se incorporan nuevos fijos y flujos ajenos a la rugosidad, pero que construyen vínculos con otras rugosidades nacionales y mundiales.

La diversificación en la educación superior abarca ahora flujos privados que compiten con los públicos. Como resultado de la inercia dinámica de esta educación, la ciencia y la tecnología se destacan en la rugosidad por su prestigio y especialización. Sin un perfil definido, la ciudad fluctúa entre el reconocimiento de la calidad de sus fijos y flujos culturales tradicionales -que construyeron su inercia dinámica histórica-, y la incorporación de otros nuevos y ajenos, en pos del progreso de la rugosidad.

En consecuencia, la construcción de un modelo urbano de la ciudad de Córdoba debería recuperar las siguientes características:

\begin{tabular}{|l|l|}
\hline \multicolumn{2}{|l|}{ Fijos por tapas Etapas históricas } \\
\hline 1. Asentamientos prehispánicos & $\begin{array}{l}\text { Los fijos_no han permanecido, pero existieron, y no tuvieron una } \\
\text { ocupación permanente }\end{array}$ \\
\hline 2. Primera fundación, 1573 & - fueron efímeros \\
\hline $\begin{array}{l}\text { 3. Segunda fundación, 1577-1770 } \\
\text { _siguieron modelos españoles, pero alcanzaron un carácter local, } \\
\text { permaneciendo en mayor medida los religiosos }\end{array}$ \\
\hline $\begin{array}{l}\text { 4. Consolidación traza fundacional, } \\
1770-1870\end{array}$ & -mantuvieron el carácter alcanzado y su uso se densificó \\
\hline $\begin{array}{l}\text { 5. Barrios-pueblo, 1880-1930 } \\
\text { 6. Industrialización y primer } \\
\text { ordenamiento urbano, 1930-1980 }\end{array}$ & $\begin{array}{l}\text { _culturales se comenzaron a perder/sustituir, incorporando otros de } \\
\text { mayor escala }\end{array}$ \\
\hline
\end{tabular}




\section{siriu. \\ SÃO PAULO $15 \sim 17$. LISBOA $25 \sim 26$ JUN 2020 \\ Seminário Internacional de Investigação em Urbanismo \\ Seminario Internacional de Investigación en Urbanismo}

7. Globalización y segundo _se reutilizaron los de valor cultural local vs. la construcción de fijos ordenamiento urbano, 1980 al de influencia global

presente

Flujos por tapas Etapas históricas

1. Asentamientos prehispánicos $\quad$ Los flujos_estuvieron marcados por el desplazamiento estacional

2. Primera fundación, $1573 \quad$ _fueron impulsados por la exploración, la religión católica y la administración colonial

3. Segunda fundación, 1577-1770 subsistieron al ser desplazados físicamente, como continuación de los anteriores se consolidaron los residenciales, administrativos, religiosos y educación superior

4. Consolidación traza fundacional, 1770-1870

_vivieron cambios inestables, y mantuvieron la especialización en educación

5. Barrios-pueblo, 1880-1930 _se diversifican los tradicionales con nuevos de residencia estacionaria-, y educación -que incluyeron avances científicos, reformas y otros actores-;

inmigración europea: mano de obra | migración: alumnos.

6. Industrialización y primer _incoporaron el flujo industrial, acompañado por la especialización ordenamiento urbano, 1930-1980 de la enseñanza superior y el comienzo de la planificación urbana. migración: alumnos

7. Globalización y segundo _fueron regulados por la planificación urbana, incorporando flujos ordenamiento urbano, 1980 al comerciales internacionales y diversificando del flujo educativo presente migración: alumnos

Rugosidades por tapas Etapas históricas

1. Asentamientos prehispánicos $\quad$ Las rugoridades_fueron aisladas, puntuales y renovables sobre el territorio natural

2. Primera fundación, 1573

3. Segunda fundación, 1577-1770 _desaparecieron

4. Consolidación traza fundacional, 1770-1870

_se insertaron en la traza fundacional, condicionadas por la religión

se acumularon sobre la traza fundacional, especializada en educación superior

5. Barrios-pueblo, 1880-1930

_se consolidaron a partir de la traza fundacional y sus barriospueblo, superando los fijos naturales y diversificando sus funciones, especializándose en ciencia y educación 


\section{SÃOPAULO15 17 LISBOA $25 \sim 26$ JUN 2020

6. Industrialización y primer _ se extendieron desde la traza, acompañando la industrialización y ordenamiento urbano, 1930-1980 la especialización en educación tecnológica

7. Globalización y segundo _se expandieron rápidamente, renovando el área central, ordenamiento urbano, 1980 al absorbiendo la especialización de la oferta educativa superior presente

\begin{tabular}{|c|c|}
\hline \multicolumn{2}{|c|}{ Inercias dinámicas por tapas Etapas históricas } \\
\hline 1. Asentamientos prehispánicos & $\begin{array}{l}\text { Las inercias dinámicas se identifican con_la agricultura y el } \\
\text { desplazamiento }\end{array}$ \\
\hline 2. Primera fundación, 1573 & $\begin{array}{l}\text { _la exploración, el control territorial y la religión católica } \\
\text { _la inmigración española-africana }\end{array}$ \\
\hline 3. Segunda fundación, 1577-1770 & _la administración, la religión católica, la educación superior \\
\hline $\begin{array}{l}\text { 4. Consolidación traza fundacional, } \\
1770-1870\end{array}$ & _la política local + ídem anterior \\
\hline 5. Barrios-pueblo, $1880-1930$ & $\begin{array}{l}\text { _ídem anterior + la Reforma 1918, la ciencia, el modelo } \\
\text { agroexportador y la inmigración europea }\end{array}$ \\
\hline $\begin{array}{l}\text { 6. Industrialización y primer } \\
\text { ordenamiento urbano, 1930-1980 }\end{array}$ & $\begin{array}{l}\text { _la industrialización, la educación superior -tecnología } \\
\text { especialización-, y la renovación y sustitución urbana } \\
\text { _la migración }\end{array}$ \\
\hline $\begin{array}{l}\text { 7. Globalización } \mathrm{y} \\
\text { ordenamiento urbano, } 1980 \text { al } \\
\text { presente }\end{array}$ & $\begin{array}{l}\text { _la educación superior -especialización - complejidad-, el } \\
\text { comercio, y la renovación, ampliación y sustitución urbana- } \\
\text { _la migración }\end{array}$ \\
\hline
\end{tabular}

Tabla. 08 Características de fijos, flujos, rugosidades e inercias dinamicas en Córdoba, según el enfoque de Milton Santos, identificadas por períodos históricos. Fuente: Elaboración propia

En síntesis, el sitio de la traza fundacional de la ciudad actúa como la rugosidad en torno a la cual fijos y flujos se organizan, consolidan, desaparecen y/o se expanden, mientras los actores se movilizan, desplazan, inmigran, migran y emigran, siguiendo la suerte de las inercias que atravesaron el territorio (exploración, conquista, administración, religión, educación superior, industrialización, comercio...). Este enunciado podría constituir una "aproximación" general al "tipo urbano" latinoamericano, aunque lo esencial sería conseguir que permita visibilizar las particularidades del ejemplo respecto al mismo.

Por eso, el territorio latinoamericano influye sustancialmente en las modificaciones que sufre el tipo urbano europeo transculturado a la región, y solo un enfoque desde el propio territorio -no solamente traza, trama y tejido-, alcanza a evidenciar las particularidades del nuevo tipo urbano, que deben ser trabajadas desde lo grafico-conceptual. 


\section{XII $\square$ SÃO PAULO15 17 LISBOA $25 \sim 26$ JUN 2020}

\section{A modo de conclusión}

Los asentamientos humanos en general -prehispánicos, hispánicos, y hasta la actualidad-, se adaptaron a las condiciones del medio geográfico. Lo hicieron modificando el territorio a partir de una traza regular y planificada, donde los espacios públicos, las viviendas, los edificios coloniales, historicistas, modernos y posmodernos son representativos de formas de vida y de modos de construcción adaptados a los recursos locales o a las posibilidades de la sociedad.

El territorio donde se asienta Córdoba posee entonces, testimonios de ocupación humana desde el periodo prehispánico, como espacio de residencia, intercambio y producción, construyendo así su espesor histórico. Sus principales rugosidades se conforman a partir de flujos como:

- la educación superior o universitaria, generada por las sedes institucionales y los barrios donde residen los estudiantes, que podría abarcar también la educación primaria y secundaria,

- la religión, que pervive en la arquitectura y en las expresiones litúrgicas tradicionales,

- la diversidad cordobesa, resultado de la confluencia de múltiples inmigraciones y migraciones, que aportaron diversidad de razas, culturas y formas de vida.

Esto sugiere que la particularidad de esta ciudad respecto a un "tipo urbano" latinoamericano, es la especialización de la rugosidad dada por la educación superior, acompañada por el comercio y la industria, a las cuales la ciencia y la tecnología siempre aportaron, y, en consecuencia, su modelo urbano debería afirmarse en esa singularidad.

En consecuencia, el modelo deseado de la forma urbana debe partir siempre de un análisis complejo y diverso del "tipo urbano" de la ciudad y su territorio, aplicando enfoques teóricos propios de la región, y con un análisis gráfico de patrones históricos que guíen la nueva planificación.

El territorio -interpretado a partir de fijos y rugosidades-, y la diversidad latinoamericana -traducida en flujos e inercias-, pueden visibilizar las múltiples identidades en la ciudad latinoamericana, gestionando inteligentemente su pasado y su futuro.

\section{Referencias bibliográficas}

ALEXANDER, C., ISHIKAWA, S., SILVERSTEIN, M. et alt. (1980 [1977]). Un lenguaje de patrones. Ciudades, edificios, construcciones. Barcelona: Gustavo Gilli,

CHUECA GOITIA, F. (1968). Breve Historia del Urbanismo. Madrid: Alianza Editorial.

FERRATER MORA, J. (1951). Diccionario de Filosofía. Buenos Aires: Editorial Sudamericana.

FOGLIA, M., GOYTIA, N., DELLAVEDOVA, D., MARICONDE, M. et al. (1988). La cuadrícula en el desarrollo de la ciudad hispanoamericana. El caso de Córdoba. Tomo I. Córdoba: Imprenta Mayor UNC.

GAMBONE, Dora., FRANCHELLO, María., MEDINA, María, CUADRADO, María, VIDAL, J., MANAVELLA, Aníbal, et al. (1996-2002). Informes Finales de Investigación Mutación y Permanencia en Córdoba. Tomo I: El Centro Urbano. Tomo II: La Nueva Córdoba, primera etapa. Tomo III: La Nueva Córdoba, Segunda Etapa". Tomo IV: Alberdi. Tomo V. General Paz. CONICOR, CONICET, SECyT. 


\section{SÃOPAULO15 17 LISBOA $25 \sim 26$ JUN 2020}

GUTIÉRREZ, R. (1992). Arquitectura y Urbanismo en lberoamérica. Madrid: Ediciones Arte Cátedra.

HARDOY, J. E. (1999). Ciudades Precolombinas. Buenos Aires. Ediciones Infinito.

KRIER, R. (1981). El Espacio Urbano. Barcelona: Gustavo Gilli.

LAGUENS, A., BONIN, M. (2012). Sociedades indígenas de las Sierras Centrales. Arqueología de Córdoba y San Luis. Córdoba: UNC.

LYNCH, K. (1959) La imagen de la ciudad. Buenos Aires: Editorial Infinito.

MARTÍ ARIS, Carlo (1990). Las variaciones de la identidad. Ensayo sobre el tipo en arquitectura. Barcelona. Ediciones del Serbal.

PANERAI, Philippe y otros. (1983 [1975]). Elementos de análisis urbano. Madrid: Instituto de Estudios de Administración Local.

QUATRÉMĖRE DE QUINCY, A. C. 2007([1792-1825]). Diccionario de arquitectura: Voces teóricas / Antoine Chrysostome Quantremère De Quincy; traducción y selección de Fernando Aliata y Claudia Schmidt. Buenos Aires: Nobuko.

RAMOS, J. L. (1997). Planificación Urbana y Regional. Teoría y Práctica. Córdoba: FAUD. UNC.

RETTAROLI J., M., EGUIGUREN, J. AlVAREZ, T.,COHEN A., A., RUBIOLI,J., et al. Los barrios pueblos de la ciudad de Córdoba. Córdoba: Ediciones Eudecor.

SANTOS, M. (1996). De la totalidad al lugar. Barcelona: Oikos-Tau. - (2000): La Naturaleza del Espacio. Técnica y Tiempo. Razón y Emoción. Madrid: Ariel. - (2004): Por otra globalización. Del pensamiento único a la conciencia universal. Bogotá: Convenio Andrés Bello. 4

SCHJETNAN, M., CALVINILLO, J., PENICHE OSORIO, M. (2004). Principios del diseño urbano/ambiental. México: Árbol Editorial. 